\title{
Differential Effects of Two Virtual Reality Interventions: Distraction Versus Pain Control
}

\author{
Desirée Loreto-Quijada, PhD, José Gutiérrez-Maldonado, $\mathrm{PhD}$, Rubén Nieto, PhD, ${ }^{2}$ \\ Olga Gutiérrez-Martínez, PhD, Marta Ferrer-García, PhD, Carmina Saldaña, PhD, \\ Adela Fusté-Escolano, $\mathrm{PhD}^{1}$, and Liudmila Liutsko, $\mathrm{PhD}^{1}$
}

\begin{abstract}
There is evidence that virtual reality (VR) pain distraction is effective at improving pain-related outcomes. However, more research is needed to investigate VR environments with other pain-related goals. The main aim of this study was to compare the differential effects of two VR environments on a set of pain-related and cognitive variables during a cold pressor experiment. One of these environments aimed to distract attention away from pain (VRD), whereas the other was designed to enhance pain control (VRC). Participants were 77 psychology students, who were randomly assigned to one of the following three conditions during the cold pressor experiment: (a) VRD, (b) VRC, or (c) Non-VR (control condition). Data were collected regarding both pain-related variables (intensity, tolerance, threshold, time perception, and pain sensitivity range) and cognitive variables (self-efficacy and catastrophizing). Results showed that in comparison with the control condition, the VRC intervention significantly increased pain tolerance, the pain sensitivity range, and the degree of time underestimation. It also increased self-efficacy in tolerating pain and led to a reduction in reported helplessness. The VRD intervention significantly increased the pain threshold and pain tolerance in comparison with the control condition, but it did not affect any of the cognitive variables. Overall, the intervention designed to enhance control seems to have a greater effect on the cognitive variables assessed. Although these results need to be replicated in further studies, the findings suggest that the VRC intervention has considerable potential in terms of increasing self-efficacy and modifying the negative thoughts that commonly accompany pain problems.
\end{abstract}

\section{Introduction}

$\mathbf{R}$ ESEARCH OVER THE LAST DECADE has shown that virtual reality (VR) is a useful tool for pain management. In general terms, VR reduces pain levels, anxiety, unpleasantness, and the need for analgesia, at the same time as increasing the pain threshold, pain tolerance, and procedural cooperation. Moreover, people enjoy using VR and are keen to use it again during other painful medical procedures. ${ }^{1-3}$

The effects of VR have been mainly explained in terms of pain distraction. Since attentional resources are limited, diverting attention away from pain by means of VR leaves fewer resources available for pain processing. ${ }^{1}$ This mechanism is not new, as distraction is a traditional psychological intervention for pain that has been shown to possess considerable efficacy. ${ }^{4}$ However, VR distraction is thought to be more effective because it is immersive and engaging, integrating many sensory experiences and, therefore, demanding a greater amount of attention. ${ }^{5}$
What is not completely clear is whether VR works solely by drawing attention away from pain. For example, it has been hypothesized that VR may also work by changing the way in which people think and perceive reality. ${ }^{6}$ Being able to identify mechanisms of action other than pain distraction, or designing interventions to act specifically on other mechanisms, could have enormous potential in terms of extending the application of VR beyond the clinical setting of acute procedural pain (where most applications have so far been applied ${ }^{7-9}$ ). Applying the strategy of pain distraction in a situation of nonacute pain outside the clinic is difficult due to the amount of equipment that is needed. However, if VR has other mechanisms of action (such as changing the way in which people think), the resulting effects could more easily be extrapolated outside the healthcare setting, thereby raising the possibility of developing useful strategies for persistent pain or long-term pain problems.

Some recent studies have used VR applications for purposes other than pain distraction. For example, Shiri et al. ${ }^{10}$

\footnotetext{
${ }^{1}$ Department of Personality, Assessment, and Psychological Treatments, University of Barcelona, Spain.

${ }^{2}$ PSiNET Research Group, Internet Interdisciplinary Institute, Universitat Oberta de Catalunya, Barcelona, Spain.
} 
designed a VR system that aimed to reinforce patients with pain-free virtual images once they achieved an adequate state of relaxation (as measured by a biofeedback procedure). Results showed that the VR system improved daily functioning and quality of life and decreased pain ratings in a sample of children with chronic headache. In another recent example, Botella et al. ${ }^{11}$ combined cognitive behavioral therapy with a VR environment that sought to develop relaxation and mindfulness skills in fibromyalgia patients. Results showed a significant reduction in pain and depression, as well as an increase in positive affect and the use of healthy coping strategies.

In order to explore whether VR might have a mechanism of action other than pain distraction, we developed and tested a VR intervention whose aim was to increase pain control. The intervention involved presenting subjects with a figure that represented pain and which could be converted into a figure representing no pain. It was hypothesized that controlling the virtual figure could help people to increase their sense of pain control. The specific aim of the present study was to test the effects of this intervention on a set of painrelated and cognitive variables, comparing the results with those obtained when using an environment designed for pain distraction.

\section{Method}

\section{Participants}

Participants were 77 psychology students at the University of Barcelona ( 70 female). They were aged between 20 and 56 years $(M=24.06 ; S D=5.22)$, and received course credits for taking part. They were all instructed to refrain from consuming alcohol or using other drugs on the day prior to the study. The exclusion criteria were cardiovascular disease, hypertension, metabolic dysfunctions, pregnancy, Raynaud's disease, epilepsy, mental disorder, chronic pain conditions, diseases producing neuropathic pain, or having taken pain/anti-inflammatory medication during the 4 hours prior to the study.

\section{Apparatus and equipment}

Cold pressor apparatus. A plastic tank $(34 \mathrm{~cm} \times 34 \mathrm{~cm} \times$ $16 \mathrm{~cm})$ filled with iced water maintained at $6^{\circ} \mathrm{C}\left( \pm 1^{\circ} \mathrm{C}\right)$ was used for the cold pressor procedure. To ensure that the water temperature remained constant before each trial, a waterproof thermometer was attached to the inside of the tank. However, the temperature could not be seen by the participant. The range of tolerance achieved with this water temperature was between 13 minutes, ${ }^{12,13}$ thereby giving participants assigned to the VR conditions enough time to interact with the VR environments.

Before each cold water immersion, an additional tank with warm water $\left(32^{\circ} \mathrm{C}\right)$ was used for stabilization of hand temperature. A digital thermometer to measure hand temperature and an atmospheric thermometer to measure room temperature were used. The room temperature was maintained at $22^{\circ} \mathrm{C}$.

Hardware. The VR environments were displayed with two BARCO ID R600 projectors controlled by a computer. They were projected onto a 2.43 meter $\times 1.82$ meter screen with a resolution of $1024 \times 768$ pixels. The distance between the screen and the participant, who was provided with StereoGraphics Corp. polarized 3D glasses, was 2 meters.
Software. The VR environments were modeled and animated with 3D Studio Max 8. Adobe Photoshop 7 was used to create the different textures. Virtools 3.5 (Educational Version) was used to program physical and visual effects, such that the participant could interact with the VR environment.

\section{Measures}

There were five pain-related measures:

Pain threshold. Pain threshold was defined as the number of seconds of immersion in the cold pressor tank until the participant reported that the cold sensation first began to feel painful.

Pain tolerance. Pain tolerance was defined as the total number of seconds that each participant kept his/her hand immersed in the cold water.

Pain sensitivity range. Pain sensitivity range (PSR) was defined as the total number of seconds that passed between the participant first reporting that the cold sensation had begun to feel painful (pain threshold) and the point at which his/ her hand was withdrawn from the cold water (pain tolerance).

Strongest pain intensity. The strongest pain intensity was assessed with a visual analog scale (VAS) that consisted of a line with two anchors: "no pain" and "the most intense pain." Immediately after withdrawal, participants were asked to rate the strength of the most intense pain they had felt during the procedure by making a vertical mark on the point of the line that they considered representative of their pain. The distance from the left anchor to the vertical mark served as the pain rating for the strongest pain intensity.

Estimation of time. Participants were asked to estimate how long they thought they had had their hand in the water by the time of withdrawal. The difference between the estimated and real time was calculated.

Two cognitive measures were used:

In vivo pain catastrophizing. This was assessed using the 13-item Pain Catastrophizing Scale $\left(\mathrm{PCS}^{14}\right)$. Subjects are asked to reflect on past episodes of pain and to rate using a 5-point scale the frequency of the catastrophic cognitions described in each item. Scores can be computed for three subscales: helplessness, rumination, and magnification. Standard PCS instructions and items were modified slightly in order to assess catastrophizing cognitions in relation to the cold water immersion (e.g., by asking subjects to consider statements such as "There was nothing I could do to reduce the intensity of the pain" or "I worried all the time about whether the pain would end"). This was done because laboratory-based studies have highlighted the importance of specifying the context in which catastrophizing is assessed. ${ }^{15-17}$ Results in our sample showed that the internal consistency was high for the in vivo PCS total score $(\alpha=0.90)$.

Pain self-efficacy. Based on the work of Bandura et al., ${ }^{18}$ pain self-efficacy was assessed through two scales: (a) perceived self-efficacy for tolerating pain, and (b) perceived self-efficacy for reducing pain intensity. The first of these 
aspects was assessed by asking participants to estimate how much time they thought they would be able to keep their hand submerged in the cold water if they had to repeat the immersion. They have to give their answer according to a series of time categories corresponding to increasing durations of immersion; the categories covered a total range from 0 seconds to 8 minutes, at 30 second intervals. The second aspectself-efficacy for reducing pain intensity-was measured using a scale that described four severities of pain (ranging from dull to excruciating). For each severity, participants were required to rate their perceived ability to reduce pain using a 3-point scale, ranging from $0=$ "limited ability" to $2=$ "good ability." A total score (ranging from 0 to 8 ) was then computed, with higher scores indicating greater perceived self-efficacy in reducing pain. The internal consistency for this scale was adequate in the present sample $(\alpha=0.78)$.

\section{Procedure}

The study was approved by the ethics committee of the University of Barcelona. A between-subjects experimental design was used. Subjects participated individually and were randomly assigned to one of the following experimental conditions: (a) Non-VR intervention (control condition), (b) VR distraction intervention, or (c) VR control enhancement intervention.

Common instructions were initially given to all participants regarding the cold pressor experiment. The experimenter explained to participants that they had to immerse their nondominant hand in the cold water up to the wrist, palm-side down, and to leave their hand open (nonfisted). Participants were instructed to say "It hurts now" when their hand began to feel uncomfortable or hurt, and "End" when they decided to withdraw their hand from the water. All participants were asked to repeat the instructions to confirm that they had understood them.

Following these common instructions, participants assigned to the VR conditions (VR distraction and VR control enhancement intervention) were provided with stereoscopic glasses and spent 2-3 minutes learning the possible interactions with the virtual environment. This was done using their dominant hand to operate the mouse. Subsequently, and for all participants regardless of their experimental group, the lights in the room were turned off, with the experimenters remaining out of sight behind the participant in order to minimize any influence their presence might have on performance. The cold pressor trial was then immediately started. For safety reasons, the maximum permitted duration of immersion was 5 minutes, although participants were unaware of this. At the end of the trial, participants were asked to rest their hand on a towel placed on the table. They were then immediately asked to complete the VAS, the in vivo PCS, and the pain self-efficacy scales based solely on their experience during the cold pressor task. The specific features of each condition were as follows.

Non-VR condition (Non-VR). Participants assigned to the Non-VR condition were told that during the immersion, they had to look at a static blank screen in front of them.

VR distraction condition (VRD). To divert the attention of participants in this condition, a virtual environment called
"Surreal World" was used. This was developed on the basis of surreal art images that were intended to surprise users with unreal objects that challenge the laws of physics. To improve the sense of presence in the surreal world, participants were able to generate simple interactions with the objects (e.g., clicking on certain objects will cause them to change or behave in another way). Participants were asked to navigate through the environment with one hand while immersing their other hand in the cold pressor.

VR control enhancement condition (VRC). For this condition, we designed a stereoscopic VR figure as a representation of pain. The figure, which appeared in the center of the screen against a black background, was an irregular sharpedged polygon that was modelled according to certain sensory descriptors (e.g., burning, cutting, sharp, stabbing, and stinging) from the McGill Pain Questionnaire. ${ }^{19}$ The figure was presented together with an unpleasant sound (a tone of $600 \mathrm{~Hz}$ at $80 \mathrm{~dB}$ ). As was explained to participants, the initial figure and the sound represented an unpleasant pain sensation, but they could be gradually manipulated to achieve a pleasant and calm state (analogous to a situation of no pain). This pleasant state was represented by a spherical figure, with a certain resemblance to natural scenery, combined with an audio track produced by a generative music engine, creating a complex series of quiet environmental sounds.

Participants were asked to try to ameliorate their pain sensation (caused by the cold pressor) by modifying the virtual figure. To modify the initial stereoscopic figure, subjects simply had to click on the right button of the mouse. Three slider controls then appeared on the screen, and these enabled participants to change the shape and color of the figure, as well as the sound. They could also rotate the figure and move it nearer or farther away by clicking and dragging the mouse.

\section{Data analysis}

All the analyses were conducted using SPSS v20. Descriptive statistics were computed for the different pain-related measures and the cognitive measures. Between-subjects univariate analyses of variance (ANOVA) were conducted to test the effects of the three different conditions (i.e., Non-VR, VRD, and VRC) on each of the pain-related and cognitive measures. Post hoc comparisons were carried out to determine the specific differences between conditions.

\section{Results}

\section{Pain-related measures}

One-way between-subjects ANOVAs revealed significant differences between the three conditions for the following variables: pain threshold, $F(2,74)=3.56, p<0.05$; pain tolerance, $F(2,74)=9.59, p<0.0001$; pain sensitivity range, $F(2$, $74)=4.11, p<0.05$, and estimation of time, $F(2,74)=3.17$, $p<0.05$. There were no statistically significant differences in relation to pain intensity, $F(2,74)=0.88, p=0.41$.

Post hoc comparisons (see Table 1) for pain threshold showed that it was significantly higher in the VRD condition compared with the Non-VR condition. None of the other comparisons showed significant differences in terms of pain threshold. As regards pain tolerance, this was significantly lower in the Non-VR condition as compared with both the 
Table 1. Effects of Interventions on Pain-Related Measures

\begin{tabular}{|c|c|c|c|c|}
\hline & Non-VR & $V R D$ & $V R C$ & Pairs comparisons [95\% CI] \\
\hline Pain threshold & $19.97(8.94)$ & $70.10(83.05)$ & $62.12(84.23)$ & $\begin{array}{l}\text { No-VR }<\text { VRD* }[-97.83,-2.43] \\
\text { VRD }>\text { VRC }[-37.77,53.73] \\
\text { VRC }>\text { No-VR }(-5.54 \div 89.85)\end{array}$ \\
\hline Pain tolerance & $47.44(20.76)$ & $145.38(101.81)$ & $148.74(114.13)$ & $\begin{array}{l}\text { No-VR }<\text { VRD } * *[-159.95,-35.93] \\
\text { VRD }<\text { VRC }(-62.83 \div 56.12) \\
\text { VRC }>\text { No-VR } * *(39.29 \div 163.30)\end{array}$ \\
\hline Pain sensitivity & $27.46(19.50)$ & $75.28(78.64)$ & $86.61(100.31)$ & $\begin{array}{l}\text { No-VR }<\text { VRD }[-99.59,3.97] \\
\text { VRD }<\text { VRC }[-61.00,38.33] \\
\text { VRC }>\text { No-VR* }(7.36 \div 110.93)\end{array}$ \\
\hline Pain intensity & $99.08(24.36)$ & $95.57(35.02)$ & $113.25(75.23)$ & $\begin{array}{l}\text { No-VR }>\text { VRD }[-31.31,38.34] \\
\text { VRD }<\text { VRC }[-38.34,31.31] \\
\text { VRC }>\text { No-VR }(-20.65 \div 49.00)\end{array}$ \\
\hline Time estimation & $-4.80(24.01)$ & $-21.92(110.53)$ & $-61.66(72.70)$ & $\begin{array}{l}\text { No-VR }>\text { VRD }[-39.42,72.40] \\
\text { VRD }>\text { VRC }[-14.91,95.66] \\
\text { VRC }<\text { No-VR } *(-112.78 \div-0.95)\end{array}$ \\
\hline
\end{tabular}

Data are mean $(S D)$.

$* p<0.05 ; * * p<0.001$.

VRD and VRC conditions, although there was no significant difference between the latter two conditions. The pain sensitivity range was significantly broader in the VRC condition in comparison with the Non-VR condition, but no significant differences were found for the other comparisons. Similarly, the underestimation of time was significantly greater in the VRC condition as compared with the Non-VR condition, but there were no significant differences for the other comparisons.

\section{Cognitive measures}

Statistically significant differences were observed in relation to self-efficacy for tolerating pain, $F(2,74)=3.15, p<0.05$, and on the Helplessness subscale of the PCS, $F(2,74)=4.62$, $p<0.05$. No differences were found for self-efficacy in re- ducing pain, $F(2,74)=0.63, p=0.53$; PCS total scores, $F(2$, $74)=1.25, p=0.29$; the Rumination subscale, $F(2,74)=0.353$, $p=0.70$; or the Magnification subscale, $F(2,74)=0.20$, $p=0.81$.

Post hoc comparisons revealed that self-efficacy for tolerating pain was significantly higher and helplessness ratings significantly lower during the VRC condition, as compared with the Non-VR condition. No significant differences emerged in any of the other comparisons (see Table 2).

\section{Discussion}

The aim of the present study was to test whether two different VR interventions, designed for pain distraction (VRD) and pain control (VRC), had differential effects on

Table 2. Effects of Interventions on Cognitive Measures Related to Pain

\begin{tabular}{|c|c|c|c|c|}
\hline & Non-VR & $V R D$ & $V R C$ & Pairs comparisons [95\% CI] \\
\hline Self-efficacy tolerance & $58.91(48.63)$ & $127.69(175.25)$ & $159.07(160.07)$ & $\begin{array}{l}\text { No-VR }<\text { VRD }[-166.49,28.93] \\
\text { VRD }<\text { VRC }[-125.17,62.41] \\
\text { VRC }>\text { No-VR* }(3.30 \div 197.02)\end{array}$ \\
\hline Self-efficacy reduction & $3.47(1.44)$ & $3.59(1.30)$ & $3.88(1.31)$ & $\begin{array}{l}\text { No-VR }<\text { VRD }[-1.03,0.80] \\
\text { VRD }<\text { VRC }[-1.17,0.58] \\
\text { VRC }>\text { No-VR }(-0.50 \div 1.32)\end{array}$ \\
\hline Total catastrophism & $18.90(8.09)$ & $15.68(11.25)$ & $14.52(9.43)$ & $\begin{array}{l}\text { No-VR }>\text { VRD }[-3.59,10.05] \\
\text { VRD }<\text { VRC }[-5.44,7.76] \\
\text { VRC }>\text { No-VR }(-11.21 \div 2.43)\end{array}$ \\
\hline Helplessness & $10.22(4.53)$ & $7.78(5.96)$ & $5.75(4.30)$ & $\begin{array}{l}\text { No-VR }>\text { VRD }[-1.12,6.01] \\
\text { VRD }>\text { VRC }[-1.45,5.52] \\
\text { VRC }>\text { No-VR** }(-8.00 \div-0.94)\end{array}$ \\
\hline Rumination & $7.45(2.50)$ & $6.86(1.89)$ & $7.04(2.71)$ & $\begin{array}{l}\text { No-VR }>\text { VRD }[-1.12,2.29] \\
\text { VRD }<\text { VRC }[-2.29,1.12] \\
\text { VRC }<\text { No-VR }(-2.10 \div 1.28)\end{array}$ \\
\hline Magnification & $2.77(2.10)$ & $2.43(2.21)$ & $2.83(2.44)$ & $\begin{array}{l}\text { No-VR }>\text { VRD }[-1.28,1.96] \\
\text { VRD }<\text { VRC }[-1.98,1.19] \\
\text { VRC }>\text { No-VR }(-1.61 \div 1.73)\end{array}$ \\
\hline
\end{tabular}

Data are mean $(S D)$.

$* p<0.05 ; * * p<0.001$. 
pain-related and cognitive variables. With respect to painrelated variables, both interventions increased pain tolerance. Additionally, the VRC intervention increased the pain sensitivity range and the degree of time underestimation, as compared with the control condition. The VRD condition significantly increased the pain threshold in comparison with the control condition. Overall, therefore, both interventions have some effects on pain-related variables, a finding that is consistent with previous studies ${ }^{1,4}$ and with our own results in earlier experiments involving the same interventions. ${ }^{20-22}$ Notably, however, neither of the two interventions had any effect on pain intensity. This result might be expected for the VRC intervention, since its objective is to increase control over pain, and similar results were obtained when using this environment in previous experiments. ${ }^{21,22}$ However, such a result is surprising in the case of the VRD intervention, since the available literature suggests that VR environments designed for pain distraction reduce pain intensity, this being the effect that we observed in a previous study that used this environment. $^{20}$ These results are likely due, at least in part, to the sample size used in the present study. However, the fact that participants were asked to indicate the strongest pain intensity rather than give an overall rating of pain intensity during the procedure may also have a bearing on these findings. ${ }^{22}$

As regards the cognitive variables, an effect on some of them was only observed for the VRC condition. This supports our hypotheses as well as the design of the intervention that aimed to enhance pain control. However, although these results are quite innovative and interesting, they now need to be replicated in larger samples, and especially in clinical contexts.

Assuming that our findings are replicated, an intervention such as the one presented here would have considerable potential in terms of extending the benefits of VR beyond the clinic. Patients would be able to use this VR intervention at the clinic to gain control over pain and change their cognitions, and this would then help them in their daily life outside the clinic. A number of reports along these lines have already been published, one example being experiments involving VR hypnosis. ${ }^{23,24}$ In the introduction to this paper, we mentioned other examples ${ }^{10,11}$ in which VR has been used for purposes other than pain distraction, and these could be particularly useful in the clinical context. What makes the intervention described here especially interesting and innovative is that it is designed to change cognitive variables (i.e., self-efficacy and catastrophizing) that have been shown to be very important in the context of chronic pain problems. ${ }^{25,26}$

The main weakness of this study is the use of an unbalanced sample regarding gender, since males and females have different responses to pain, ${ }^{27}$ and this may influence the final results. Replications of this study with a more balanced distribution of males and females are needed. Also, in contrast to previous studies on VRD, this study used nonimmersive VR. The link between immersion and presence and between pain distraction and presence is well established, so this difference may explain the limited efficacy of VRD in our study. The results obtained in this study showing that the intervention designed to enhance control (VRC) has a greater effect on the cognitive variables assessed than the intervention designed to increase distraction (VRD) can be applied, at this moment, only to nonimmersive VR systems. Replications of this study with immersive VR are needed.

\section{Acknowledgment}

This research was supported by Fundació La Marató de TV3.

\section{Author Disclosure Statement}

No competing financial interests exist.

\section{References}

1. Li A, Montaño Z, Chen VJ, et al. Virtual reality and pain management: current trends and future directions. Pain Management 2011; 1:147-157.

2. Shahrbanian S, Ma X, Korner-Bitensky N, et al. Scientific evidence for the effectiveness of virtual reality for pain reduction in adults with acute or chronic pain. Studies in Health Technology \& Informatics 2009; 144:40-43.

3. Rutter CE, Dahlquist LM, Weiss KE. Sustained efficacy of virtual reality distraction. Journal of Pain 2009; 10:391-397.

4. Malloy KM, Milling LS. The effectiveness of virtual reality distraction for pain reduction: a systematic review. Clinical Psychology Review 2010; 30:1011-1018.

5. Mahrer NE, Gold JI. The use of virtual reality for pain control: a review. Current Pain \& Headache Reports 2009; 13:100-109.

6. Keefe FJ, Huling DA, Coggins MJ, et al. Virtual reality for persistent pain: a new direction for behavioral pain management. Pain 2012; 153:2163-2166.

7. Hoffman HG, Chambers GT, Meyer WJ, et al. Virtual reality as an adjunctive non-pharmacologic analgesic for acute burn pain during medical procedures. Annals of Behavioral Medicine 2011; 41:183-191.

8. Kipping B, Rodger S, Miller K, et al. Virtual reality for acute pain reduction in adolescents undergoing burn wound care: a prospective randomized controlled trial. Burns 2012; 38:650-657.

9. Wohlheiter KA, Dahlquist LM. Interactive versus passive distraction for acute pain management in young children: the role of selective attention and development. Journal of Pediatric Psychology 2013; 38:202-212.

10. Shiri S, Feintuch U, Weiss N, et al. A virtual reality system combined with biofeedback for treating pediatric chronic headache-a pilot study. Pain Medicine 2013; 14:621-627.

11. Botella C, Garcia-Palacios A, Vizcaino Y, et al. Virtual reality in the treatment of fibromyalgia: a pilot study. Cyberpsychology, Behavior, \& Social Networking 2013; 16:215-223.

12. Mitchell LA, MacDonald RA, Brodie EE. Temperature and the cold pressor test. Journal of Pain 2004; 5:233-237.

13. Piira T, Hayes B, Goodenough B, et al. Effects of attentional direction, age, and coping style on cold-pressor pain in children. Behavior Research Therapy 2006; 44:835-848.

14. Sullivan MJL, Bishop SR, Pivik J. The Pain Catastrophizing Scale: development and validation. Psychological Assessment 1995;7:524-532.

15. Dixon KE, Thorn BE, Ward LC. An evaluation of sex differences in psychological and physiological responses to experimentally-induced pain: a path analytic description. Pain 2004; 112:188-196.

16. Edwards RR, Campbell CM, Fillingim RB. Catastrophizing and experimental pain sensitivity: only in vivo reports of catastrophic cognitions correlate with pain responses. Journal of Pain 2005; 6: 338-339.

17. Edwards RR, Smith MT, Stonerock G, et al. Pain-related catastrophizing in healthy women is associated with greater 
temporal summation of and reduced habituation to thermal pain. The Clinical Journal of Pain 2006; 22:730-737.

18. Bandura A, O'Leary A, Taylor CB, et al. Perceived selfefficacy and pain control: opioid and nonopioid mechanisms. Journal of Personality \& Social Psychology 1987; 53:563-571.

19. Melzack R. The McGill Pain Questionnaire: major properties and scoring methods. Pain 1975; 1:277-299.

20. Gutierrez-Maldonado J, Gutierrez-Martinez O, CabasHoyos K. Interactive and passive virtual reality distraction: effects on presence and pain intensity. Studies in Health Technology \& Informatics 2011; 167:69-73.

21. Gutiérrez-Maldonado J, Gutiérrez-Martínez O, LoretoQuijada D, et al. The use of virtual reality for coping with pain with healthy participants. Psicothema 2012; 24:516522.

22. Loreto-Quijada D, Gutiérrez-Maldonado J, GutiérrezMartínez O, Nieto R. Testing a virtual reality intervention for pain control. European Journal of Pain 2013; 17:14031410.

23. Soltani M, Teeley AM, Wiechman SA, et al. Virtual reality hypnosis for pain control in a patient with gluteal hidradenitis: a case report. Contemporary Hypnosis \& Integrative Therapy $2011 ; 28: 142-147$.
24. Teeley AM, Soltani M, Wiechman SA, et al. Virtual reality hypnosis pain control in the treatment of multiple fractures: a case series. American Journal of Clinical Hypnosis 2012; 54:184-194.

25. Gatchel RJ, Okifuji A. Evidence-based scientific data documenting the treatment and cost-effectiveness of comprehensive pain programs for chronic nonmalignant pain. The Journal of Pain 2006; 7:779-793.

26. Keefe FJ, Rumble ME, Scipio CD, et al. Psychological aspects of persistent pain: current state of the science. The Journal of Pain 2004; 5:195-211.

27. Wiesenfeld-Hallin Z. Sex differences in pain perception. Gender Medicine 2005; 2:137-145.

Address correspondence to: Dr. José Gutiérrez-Maldonado Department of Personality, Assessment, and Psychological Treatments University of Barcelona

Passeig Vall d'Hebrón, 171 08035 Barcelona Spain

E-mail: jgutierrezm@ub.edu 